\title{
FAITHFUL NOETHERIAN MODULES
}

\author{
EDWARD FORMANEK ${ }^{1}$
}

\begin{abstract}
The Eakin-Nagata theorem says that if $T$ is a commutative Noetherian ring which is finitely generated as a module over a subring $R$, then $R$ is also Noetherian. This paper proves a generalization of this result. However, the main interest is that the proof is very elementary and uses little more than the definition of "Noetherian".
\end{abstract}

All rings are associative and have a unit, subrings have the same unit, and modules are unitary.

A theorem due independently to Eakin [2] and Nagata [7] says that if $T=R a_{1}+\cdots+R a_{k}$ is a commutative ring finitely generated as a module over a subring $R$, then $R$ is Noetherian if $T$ is Noetherian. A later proof was given by Mollier [6] and there have been noncommutative generalizations by Eisenbud [3], Björk [1] and Jategaonkar and Formanek [4].

The object of this paper is to present a simple and elementary proof of the Eakin-Nagata theorem which generalizes the original version in a new direction. The proof is essentially a contraction of Eakin's proof as presented by Kaplansky in [5, Exercises 14-15, p. 54], based on the observation that much of that proof disappears if one is not "handicapped" by the hypothesis that $T$ is a ring. More precisely, $T$ is viewed as an $R$ module and the Eakin-Nagata theorem is viewed as a generalization of the basic result that a commutative ring which has a faithful Noetherian module is itself Noetherian [5, Exercise 10, p. 53].

THEOREM. Let $R$ be a commutative ring and $T=R a_{1}+\cdots+R a_{k} a$ faithful finitely generated left $R$-module which satisfies the ascending chain condition on "extended submodules" $A T$, where $A$ is an ideal in $R$. Then $T$ is a Noetherian $R$-module and hence $R$ is a Noetherian ring.

Proof. Suppose conversely that $T$ is not a Noetherian $R$-module.

Step I. Let $A T$ be an extended submodule of $T$ maximal with respect to the property: $T / A T$ is not a Noetherian $R$-module. Then $R / \operatorname{Ann}(T / A T)$ and $T / A T$ satisfy the hypothesis of the theorem, but $T / A T$ is not

Received by the editors March 6, 1973.

AMS (MOS) subject classifications (1970). Primary 13E05.

Key words and phrases. Eakin-Nagata theorem, ascending chain condition.

1 The author is a Canadian NRC postgraduate fellow supported by grant A7171.

(c) American Mathematical Society 1973 
Noetherian. Hence we may replace $R$ by $R / \operatorname{Ann}(T / A T)$ and $T$ by $T / A T$ and thus assume that $T / B T$ is Noetherian whenever $B T$ is a nonzero extended submodule of $T$.

Step II. Suppose $U$ is any submodule of $T$. Then $T / U$ is a faithful $R$-module iff for each nonzero $r \in R$ there is at least one of $a_{1}, \cdots, a_{k}$ (depending on $r$ ) such that $r a_{i} \notin U$. This latter property is inductive, so $T$ has a maximal submodule $U$ with respect to the property: $T / U$ is a faithful $R$-module.

Now $R$ and $T / U$ satisfy the hypotheses of the theorem. If $T / U$ were Noetherian then $R$ would be Noetherian, since $T / U$ is a faithful $R$-module, and then $T$ would be Noetherian, a contradiction. Hence $T / U$ is not Noetherian, and we may replace $T$ by $T / U$.

Step III. Summarizing Steps I and II, we may assume that

(1) $T$ is not a Noetherian $R$-module.

(2) If $A T$ is a nonzero extended submodule of $T, T / A T$ is a Noetherian $R$-module.

(3) If $U$ is a nonzero submodule of $T, T / U$ is not a faithful $R$-module. Now suppose $U$ is any nonzero submodule of $T$. T/U is not a faithful $R$-module so there is a nonzero $r \in R$ such that $r T \subseteq U$. $r T$ is an extended submodule so $T / r T$ is Noetherian and thus $T / U$ is Noetherian. Hence $T$ is Noetherian, since every proper quotient of $T$ is Noetherian and this contradiction completes the proof.

If $T$ is a ring (not necessarily commutative) and $A$ is an ideal of $R$, then $A T$ is the right ideal generated by $A$, and if $R$ is central in $T$, then $A T$ is the two-sided ideal of $T$ generated by $A$. Thus the theorem yields noncommutative generalizations of the original Eakin-Nagata theorem. These are stated below and are due to Björk, who proved the above theorem with the additional hypothesis that $T$ is a ring.

COROLlARY (BJöRK [1]). Suppose $T=R a_{1}+\cdots+R a_{k}$ is a ring, where $R$ is a commutative subring of $T$.

(1) If $T$ satisfies ACC on extended right ideals, then $R$ is Noetherian.

(2) If $R$ is central in $T$ and $T$ satisfies ACC on extended two-sided ideals, then $R$ is Noetherian.

Björk has asked whether $R$ is Noetherian if $T$ is left Noetherian. This is proved in [4] using the theory of polynomial identity rings, and the theorem of this paper also plays a role.

\section{REFERENCES}

1. J. E. Björk, On Noetherian and Artinian chain conditions of associative rings, Arch. Math. (Basel) (to appear). 
2. P. M. Eakin, Jr., The converse to a well-known theorem on Noetherian rings, Math. Ann 177 (1968), 278-282. MR 37 \#1360.

3. D. Eisenbud, Subrings of Artinian and Noetherian rings, Math. Ann. 185 (1970), 247-249. MR 41 \#6885.

4. E. Formanek and A. V. Jategaonkar, Subrings of Noetherian rings (preprint).

5. I. Kaplansky, Commutative rings, Allyn and Bacon, Boston, Mass., 1970. MR 40 \#7234.

6. D. Mollier, Descente de la propriété noethérienne, Bull. Sci. Math. 94 (1970), 25-31. MR 42 \#4533.

7. M. Nagata, A type of subrings of a Noetherian ring, J. Math. Kyoto Univ. 8 (1968), 465-467. MR 38 \#4460.

Department of Mathematics, Carleton University, Ottawa, Ontario, Canada

Current address: School of Mathematics, Institute for Advanced Study, Princeton, New Jersey 08540 